\title{
Ética docente y del cuidado en la educación
}

Teaching and care ethics in education Ensino e cuidado da ética na educação

\author{
Guadalupe Ibarra Rosales \\ Universidad Nacional Autónoma de México, México \\ irge@unam.mx \\ https://orcid.org/0000-0002-2180-357X
}

\section{Resumen}

Introducción: Este artículo se enfoca en el estudio de la ética docente, para lo cual se tomaron en cuenta los resultados de una encuesta sobre la ética de la docencia y de la enseñanza aplicada a un grupo de alumnos de la carrera de Pedagogía de la Universidad Nacional Autónoma de México. Objetivo: Conocer la ética docente en la práctica educativa a través de los rasgos y las características que en opinión de los alumnos encuestados califican a un buen docente. Método: La estrategia metodológica fue una encuesta que tuvo como instrumento un cuestionario con preguntas abiertas. La codificación de los resultados se efectuó con base en categorías elaborados para comprender y analizar la información recabada. Los referentes teóricos de este estudio fueron la concepción de la docencia como una profesión de ayuda y los planteamientos centrales de la ética del cuidado, los cuales permitieron interpretar los hallazgos desde estas perspectivas teóricas. Resultados: Se muestra que en un buen docente están presentes dos dimensiones: la pedagógica (25\%) y la ética $(75 \%)$. Se destaca que la dimensión ética fue colocada por encima de la dimensión pedagógica. El análisis de la dimensión pedagógica expone que para estos alumnos son relevantes y prioritarias las capacidades pedagógicas y didácticas para la enseñanza (44 \%). En la dimensión ética los alumnos situaron en primer término a las actividades pedagógicas y educativas de ayuda y apoyo para el aprendizaje de los alumnos (48 \%). Conclusión: De los resultados obtenidos se desprende que en la ética docente se integran estas dos 


\section{Revista Iberoamericana \\ de las Ciencias Sociales y \\ Humanísticas}

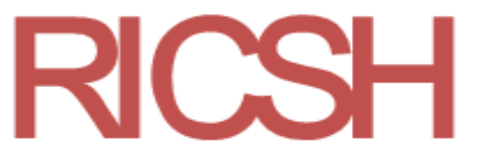

ISSN: 2395 - 7972

dimensiones. La pedagógica expresa la competencia profesional del docente que lo faculta para llevar a cabo el proceso de enseñanza y aprendizaje, mientras que la dimensión ética da cuenta de las prácticas educativas y pedagógicas realizadas con un sentido ético, es decir, para favorecer el aprendizaje de los alumnos.

Palabras clave: buen docente, ética del cuidado, ética docente, práctica docente, valores.

\section{Abstract}

Introduction: this article includes a study of teaching ethics, the empirical support of which are the results of a survey on the ethics of teaching and teaching carried out on a group of students of the Pedagogy career at UNAM. Objective: to know the teaching ethics in educational practice through the features and characteristics that in the opinion of the surveyed students qualify a good teacher. Method: the methodological strategy was a survey that had as an instrument a questionnaire with open questions. The coding of the results was carried out based on categories developed to understand and analyze these results. The theoretical references of this study were the conception of teaching as a helping profession and the central approaches to the ethics of care, which allowed the results to be interpreted from these theoretical perspectives. Results: it is shown that two dimensions are present in a good teacher: pedagogical $(25 \%)$ and ethics (75\%). It is noted that the ethical dimension was placed above the pedagogical dimension. The analysis of the pedagogical dimension shows that for these students the pedagogical and didactic capacities for teaching are relevant and priority (44\%). In the ethical dimension, the students placed in the foreground the pedagogical and educational activities to help and support the students' learning (48\%). Conclusion: the results obtained show that these two dimensions are integrated in teaching ethics. The pedagogical expresses the professional competence of the teacher that empowers him to carry out the teaching and learning process. While the ethical dimension accounts for the educational and pedagogical practices carried out with an ethical sense, that is, to favor the learning of students.

Keywords: good teacher, care ethics, teaching ethics, teaching practice, values. 


\section{Revista Iberoamericana \\ de las Ciencias Sociales y \\ Humanísticas}

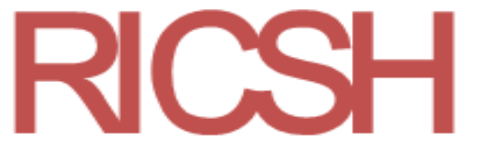

ISSN: $2395-7972$

\section{Resumo}

Introdução: Este artigo enfoca o estudo da ética docente, para o qual foram considerados os resultados de uma pesquisa sobre a ética do ensino e do ensino aplicada a um grupo de alunos da carreira de Pedagogia da Universidade Nacional Autônomo do México. Objetivo: Conhecer o ensino da ética na prática educacional através dos traços e características que na opinião dos alunos pesquisados qualificam um bom professor. Método: A estratégia metodológica foi uma pesquisa que teve como instrumento um questionário com questões abertas. A codificação dos resultados foi realizada com base em categorias desenvolvidas para compreender e analisar as informações coletadas. O referencial teórico deste estudo foi a concepção da docência como profissão auxiliar e as abordagens centrais da ética do cuidado, o que nos permitiu interpretar os achados a partir dessas perspectivas teóricas. Resultados: Mostra-se que duas dimensões estão presentes em um bom professor: pedagógica $(25 \%)$ e ética $(75 \%)$. Ressalta-se que a dimensão ética foi colocada acima da dimensão pedagógica. A análise da dimensão pedagógica mostra que para estes alunos as capacidades pedagógicas e didáticas para o ensino são relevantes e prioritárias (44\%). Na dimensão ética, os alunos colocaram em primeiro plano as atividades pedagógicas e educacionais de ajuda e apoio à aprendizagem dos alunos (48\%). Conclusão: Os resultados obtidos mostram que essas duas dimensões estão integradas no ensino da ética. A pedagógica expressa a competência profissional do professor que o capacita para realizar o processo de ensino e aprendizagem, enquanto a dimensão ética dá conta das práticas educacionais e pedagógicas realizadas com sentido ético, ou seja, para favorecer a aprendizagem dos alunos. alunos.

Palavras-chave: bom professor, ética do cuidado, ética do professor, prática docente, valores.

Fecha Recepción: Julio 2020

Fecha Aceptación: Diciembre 2020 


\section{Revista Iberoamericana \\ de las Ciencias Sociales y \\ Humanísticas}

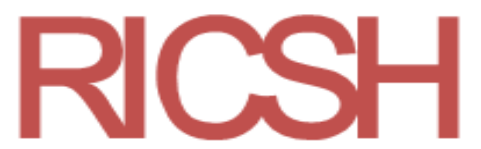

ISSN: $2395-7972$

\section{Introducción}

En este artículo se analizan los resultados de una encuesta sobre la ética de la docencia y de la enseñanza en la cual participaron alumnos de la licenciatura en Pedagogía de la Universidad Nacional Autónoma de México (UNAM). Este tema ha sido estudiado por diversos autores (Alonso, 2019; Cabalín, Navarro, Zamora y San Martín, 2010; Casero, 2010; Corona, 2008; Giné, 2009), los cuales han señalado las competencias, habilidades, atributos

profesionales y personales que, según la opinión de los alumnos, caracterizan a un buen docente.

El estudio que se presenta en este artículo concibe al buen docente desde una perspectiva ética. Este giro conceptual consiste en analizar — con el enfoque de la ética docente - las características, los rasgos y los atributos de un buen docente. El fundamento teórico de esta visión del buen docente se sustenta en la concepción de las profesiones elaborada por Hortal (2002), quien plantea que en todo profesional es posible que se integre un buen profesional y un profesional bueno.

Un buen profesional es una persona capaz y competente que cuenta con los conocimientos científicos y las habilidades profesionales para proporcionar un servicio eficaz, mientras que un profesional bueno asume la responsabilidad y el compromiso de contribuir al bienestar de la sociedad a través del servicio prestado. Esta visión ética de las profesiones permite comprender que un buen profesional no es solo como aquel que tiene un comportamiento moral o se conduce conforme a los valores, sino que alude a la excelencia en la prestación del servicio y al bien que aporta a la sociedad.

En concordancia con esta idea, García y García (2012) — al analizar la excelencia en la práctica profesional - muestran de manera clara los aspectos que comprenden a un buen profesional. En tal sentido, cuando se emplea el adjetivo profesional para calificar a un trabajo, se hace énfasis en que su realización alcanza cierto grado de "excelencia” tanto en el sentido de hacer bien el trabajo (perspectiva técnica) como en el de hacer el bien con el trabajo (sentido ético o moral), pues beneficia a los destinatarios de la tarea y al mismo profesional que la ejecuta (García y García, 2012).

Por ello, este estudio considera que la ética docente no se reduce a los códigos éticos y deontológicos que han elaborado los colectivos docentes para prescribir normas y deberes morales enfocados en orientar la realización de la práctica docente. Por el contrario, se considera que esta ética se está construyendo a través de la realización de la práctica docente, 


\section{Revista Iberoamericana \\ de las Ciencias Sociales y \\ Humanísticas}

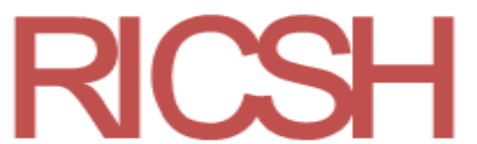

ISSN: 2395 - 7972

donde es posible reconocer aquellas labores que han logrado o aspiran a la excelencia tanto en su competencia profesional como en el sentido ético. Esta visión se sustenta en la concepción de la ética docente elaborada por Martínez (2010), quien la entiende como una ética aplicada, pero no acabada, sino abierta a las aportaciones que pueden hacer los propios docentes y los profesionales de la educación, otros profesionales en los que han incidido la práctica de la docencia, los padres de familia, los alumnos, así como la sociedad en su conjunto.

Como se puede advertir, este enfoque reconoce a los alumnos como uno de los sujetos educativos que pueden contribuir a definir y construir la ética docente, ya que son los directamente afectados de manera positiva o negativa. Por ello, este trabajo tuvo como estrategia metodológica la realización de una encuesta a alumnos perfilada a conocer los rasgos y las características que a su juicio definen a un buen docente.

Esta idea obligó a recuperar la concepción de la docencia como una profesión de ayuda, la cual ha sido desarrollada por García (1998), Altarejos (2003) y García y García (2012). Para estos autores la docencia como profesión no se limita solo a cumplir la función de transmitir conocimientos con fines de aprendizaje, sino también a apoyar y colaborar con el alumno en su proceso de aprendizaje. Esta visión es la que posibilita concebir las actividades de apoyo y acompañamiento por parte del docente como acciones de cuidado y atención al alumno propias de la ética del cuidado. En consecuencia, los referentes teóricos de este estudio son los siguientes: la concepción de la docencia como una profesión de ayuda y los planteamientos centrales de la ética del cuidado con base en los cuales fue posible comprender y analizar los resultados de la encuesta desde la perspectiva de la ética docente.

Según los resultados recabados con la encuesta, en un buen docente se integran dos dimensiones: la pedagógica y la ética. La dimensión pedagógica comprende las competencias y capacidades pedagógicas y didácticas que requiere tener un docente para realizar la enseñanza, mientras que la dimensión ética incluye las acciones y actitudes que puede tener el docente para apoyar el aprendizaje de los alumnos.

La ética del cuidado constituye el horizonte conceptual con base en el cual se analizaron los resultados de la encuesta correspondientes a la dimensión ética de un buen docente. Desde esta perspectiva fue posible reconocer y analizar las actitudes y acciones de apoyo y acompañamiento que los alumnos consideran que es deseable que tenga un buen docente. Asimismo, los planteamientos de esta ética aportan elementos de la enseñanza que 


\section{Revista Iberoamericana \\ de las Ciencias Sociales y \\ Humanísticas}

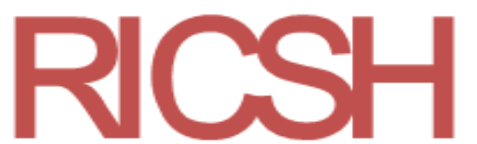

ISSN: $2395-7972$

se pueden articular en la práctica docente para realizarse como una práctica de apoyo y ayuda para los alumnos.

\section{Referentes teóricos}

\section{La docencia como una profesión de ayuda}

En el campo de estudio de la docencia es posible reconocer dos enfoques que no se contraponen, sino que se complementan. La primera perspectiva (García, Jover, Escámez, 2010; Martínez, 2010) concibe a la docencia como una profesión (ya que cuenta con los atributos que permiten reconocerla como tal), mientras que la segunda la entiende como una profesión de ayuda (Altarejos, 2003; García, 1998; García y García, 2012).

El enfoque sociológico de las profesiones ha establecido que las profesiones modernas se definen porque cuentan con ciertos rasgos y características que las distinguen: por ejemplo, tener una formación profesional adquirida en una institución de educación superior, contar con conocimientos científicos y tecnológicos especializados, formar parte de una asociación o colegio profesional, prestar un servicio a la sociedad y tener un código deontológico o ético (Cortina, 2000).

En contraste, desde la mirada pedagógica, Altarejos (2003) explica que la docencia es una profesión de ayuda, ya que se caracteriza por el apoyo que el docente brinda al alumno en el proceso educativo. Para dicho autor, este es un rasgo sustantivo de su profesionalidad, pues contribuye a que el mismo alumno desarrolle sus potencialidades educativas. Desde esta perspectiva, la ayuda se puede comprender como un rasgo de la profesionalidad del docente porque la función del docente no se limita a la simple transmisión de los conocimientos, sino que también es su facultad colaborar con el alumno para que logre el aprendizaje esperado. Esta tarea propia del docente la puede realizar en la medida en que la relación educativa se comprenda y desarrolle como una relación de ayuda. En este punto, Atarejos (2003) precisa lo siguiente:

La relación de ayuda se establece como co-operación; como apoyo o asistencia para que el aprendiz procure algo por sí mismo, algo que puede y debe obtener por sí mismo; pero que se favorece y se propicia con la ayuda de otro, que es experto en la asistencia a tal logro (...); en la ayuda el destinatario es alguien reforzado en su propia acción, y dicho refuerzo es precisamente el bien que se ofrece; el ayudado es un agente activo (p. 43). 


\section{Revista Iberoamericana \\ de las Ciencias Sociales y \\ Humanísticas}

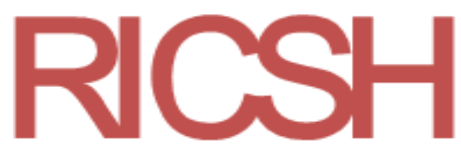

ISSN: $2395-7972$

Altarejos (2003) al tomar distancia de la concepción sociológica de las profesiones muestra que el carácter profesional de la docencia no solo se sustenta en los atributos clásicos establecidos por la perspectiva sociológica mencionada con anterioridad, porque estos rasgos y características se establecen en relación con el servicio que deben proporcionar las profesiones modernas a la sociedad. Este autor considera, por ende, que la docencia ofrece — más que un servicio - un bien que consiste en impulsar y propiciar la realización del aprendizaje por el mismo alumno. Por ello, se define como una profesión de ayuda a la que le corresponden otros atributos que no se han considerado, como "competencia, iniciativa, responsabilidad, compromiso y dedicación” (Altarejos, 2003, p. 44).

García y García (2012) coinciden con este planteamiento, porque también conciben la relación educativa como un vínculo de ayuda asimétrico, donde el alumno representa el sujeto vulnerable en términos educativos. Los autores argumentan esa asociación asimétrica en los siguientes términos:

Una de las principales características de las profesiones de ayuda educativa es que en ellas se establecen relaciones de tipo asimétrico, porque el educador y el educando no se sitúan — desde la perspectiva formativa - en un plano de igualdad, sino una de las partes se presenta como dependiente de la otra: el educando aparece como la parte pedagógicamente más débil y vulnerable en la relación (García y García, 2012, pp. 16- 17).

Así, el proceso educativo coloca al docente en un nivel superior ante el alumno debido a que tiene un conjunto de atributos que lo invisten de autoridad y poder, es decir, posee y domina los conocimientos que transmite y cuenta con edad y experiencia superior al alumno. En otras palabras, el mismo proceso educativo sitúa al alumno en condición de dependencia, ya que tiene la necesidad de adquirir los conocimientos que no tiene y requiere del apoyo del docente para realizar su aprendizaje (García, 1998; Ibarra, 2018).

Por ello, García (1998) concibe al docente como un agente moral, ya que la asimetría propia de la relación educativa exige actitudes y comportamientos éticos imprescindibles para proporcionar el apoyo que requiere el alumno: 


\section{Revista Iberoamericana \\ de las Ciencias Sociales y \\ Humanísticas}

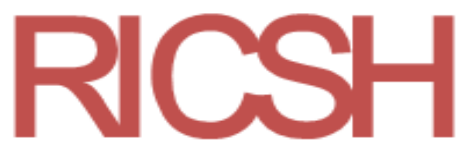

ISSN: 2395 - 7972

La única posibilidad de un comportamiento ético del profesor frente a su alumno es, por tanto, la solicitud, el cuidado y el cariño que tiene como finalidad propia el conseguir que el alumno pueda prescindir del apoyo que le ha proporcionado para su proceso de maduración personal (García, 1998, p. 298).

A partir de las reflexiones anteriores surgen interrogantes con estas: ¿cómo puede proporcionar el docente la ayuda que demanda el alumno?, ¿qué actitudes y comportamientos éticos involucra este apoyo? Una alternativa de respuesta se halla en los planteamientos de la ética del cuidado.

\section{La ética del cuidado en la educación}

En sintonía con la concepción de la docencia como una profesión de ayuda, la ética del cuidado toma en cuenta la situación de vulnerabilidad del alumno en el proceso educativo. Con base en esto, desarrolla una propuesta pedagógica y moral para que el docente mediante su práctica educativa pueda brindar a los alumnos la atención, el cuidado y el apoyo que necesitan para alcanzar sus aprendizajes.

Por eso, Noddings (2009) pone en el centro del proceso educativo las necesidades y carencias de tipo pedagógico y afectivo que tiene el alumno para mostrar la importancia del apoyo y del cuidado del docente en el proceso de enseñanza y aprendizaje. Para esta autora el cuidado (caring) es "el compromiso a prestar atención y la disposición a responder de modo favorable a las legítimas necesidades del otro" (Noddings, 1995, citada por Weis, 2007, p. 30).

El cuidado se comprende entonces como la responsabilidad y el compromiso que le corresponde asumir al docente ante la demanda de apoyo por parte del alumno. Este es el fundamento moral para comprender el cuidado como una práctica ética, puesto que el docente tiene la opción de permanecer indiferente ante las necesidades del alumno o puede responder brindándole la atención y los recursos pedagógicos y educativos para solventar sus carencias educativas.

Para Noddings (2009) el aula constituye el núcleo del desarrollo de la práctica del cuidado porque es el espacio en el cual se realiza el proceso educativo donde los alumnos pueden manifestar las necesidades y carencias educativas que tienen. Por ello, la ética del cuidado se asume como una ética relacional (Noddings, 2009). En este marco, para esta 


\section{Revista Iberoamericana \\ de las Ciencias Sociales y \\ Humanísticas}

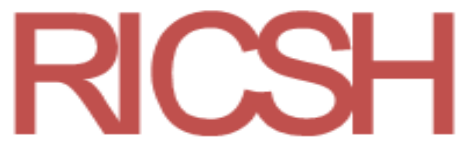

ISSN: 2395 - 7972

perspectiva la relación educativa es clave para dar cabida a la ética del cuidado, porque la atención y la ayuda que puede prestar el docente dependen en gran medida del tipo de relación educativa que establezca con los alumnos.

Con base en este enfoque, para la ética del cuidado, la relación educativa puede configurarse en el proceso educativo como una relación de cuidado donde el docente ponga al servicio de los alumnos sus conocimientos y experiencia porque cuenta con las condiciones para ofrecer ese apoyo de manera gratuita y desinteresada. Para esta ética, la relación de cuidado es un vínculo interpersonal entre el docente y el alumno que involucra el intercambio, el diálogo y la comunicación (Noddings, 2009). De este modo el docente puede conocer las necesidades, deseos y proyectos de los alumnos para ofrecer una respuesta concreta.

Mesa (2004) precisa que la relación docente-alumno configurada como una relación de cuidado no debe confundirse ni asemejarse a la relación madre-hijo o padre-hijo. Por eso, recupera lo establecido por Noddings, quien señala lo siguiente: "Yo no necesito establecer una relación profunda duradera que implica mucho tiempo con cada estudiante. Lo que debo hacer es estar presente de manera total y no selectiva cuando cada uno de ellos se dirige a mí” (Noddings, 1984, citada por Mesa, 2004, p 180).

Comins (2017) entiende la ética del cuidado en educación como una pedagogía del cuidar, y considera que es un proyecto educativo novedoso y diferente que tiene un nivel macro y uno micro, ambos presentes en el aula. Esta autora precisa que para Noddings los dos niveles representan dos dimensiones del cuidado: "Es un fin de la enseñanza (contenido curricular, nivel macro) y también un medio (método pedagógico, nivel micro)" (Comins, 2017, p. 153).

El presente artículo toma en cuenta el nivel micro y delimita el análisis a la práctica docente en el aula, lo que - como ya se indicó - es el corazón de la ética del cuidado. Esto se fortalece con el argumento de Comins (2017): "El aula debe ser el ejemplo, microcosmos, de esa relación atenta y cuidadosa que queremos ver en el mundo. El cuidado en el aula es una forma de estar en relación, que persigue enseñar un modo-de-ser-en-el-mundo, el modode-ser-cuidado" (Comins, 2017, pp. 153-154).

Sin embargo, es importante señalar que la ética del cuidado en términos morales es más que un método pedagógico porque no se reduce a estrategias y técnicas pedagógicas y didácticas, sino que constituye una práctica ética que realiza el docente a favor del alumno 


\section{Revista Iberoamericana \\ de las Ciencias Sociales y \\ Humanísticas}

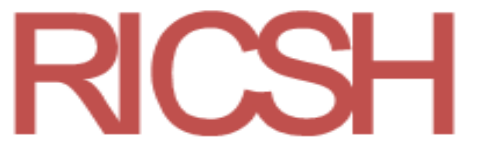

ISSN: 2395 - 7972

para impulsar el crecimiento y el desarrollo de sus potencialidades tanto educativas como morales.

En términos pedagógicos, la ética del cuidado es una práctica educativa realizada con un sentido ético porque en el proceso de enseñanza-aprendizaje el docente asume su responsabilidad ante las necesidades educativas de los alumnos y les proporciona los conocimientos, habilidades y capacidades para cubrir estas carencias y alcanzar un aprendizaje significativo. Es decir, la ética del cuidado está perfilada para lograr la formación integral del alumno, y no solo la dimensión moral, por lo que representa una forma diferente de concretar la educación, que se distingue porque el proceso de enseñanza-aprendizaje se lleva a cabo a través de la práctica del cuidado, entendida como las acciones educativas de apoyo y acompañamiento que brinda el docente al alumno.

\section{Metodología}

La presente fue una investigación exploratoria de corte cuantitativo. La metodología usada fue la encuesta, la cual se aplicó a diferentes grupos de estudiantes en distintos periodos (en este artículo se detallan los resultados obtenidos de un solo grupo de alumnos encuestados). La encuesta como metodología se fundamenta en los señalamientos de LópezRoldán y Fachelli (2015), quienes explican que la encuesta es una metodología de investigación porque comprende un conjunto de etapas y procedimientos articulados de manera coherente para obtener información científica. El propósito no fue conocer las causas o factores que inciden en la ética docente, sino recabar la opinión de los alumnos sobre las características de un buen docente. Esta encuesta tuvo como instrumento o técnica para recabar la información un cuestionario con preguntas abiertas, las cuales permitieron obtener información cualitativa sobre los rasgos de un buen docente. Por ende, el objetivo particular de la encuesta fue conocer los aspectos y elementos que toman en cuenta los alumnos encuestados como rasgos o características de un buen docente. En síntesis, la metodología de investigación comprendió las siguientes etapas:

a. Diseño y elaboración del cuestionario con preguntas abiertas para elaborar una encuesta sobre la ética de la docencia y de la enseñanza. El cuestionario se estructuró con cinco preguntas abiertas, aunque en este trabajo se presentan los resultados de dos de ellas: 1) cinco rasgos de un buen docente (Hirsch, 2005) y 2) cinco valores para la formación docente. Estas preguntas ofrecen resultados para conocer la ética 


\section{Revista Iberoamericana \\ de las Ciencias Sociales y \\ Humanísticas}

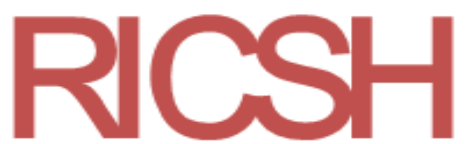

ISSN: $2395-7972$

docente a través de las características y atributos de un buen docente, así como los valores deseables para el futuro profesor.

b. Elección de la muestra de estudiantes para aplicar el cuestionario. El criterio central para definir la muestra fue el siguiente: alumnos que tuvieran conocimientos de pedagogía y educación, pues de ese modo podían determinar los rasgos de un buen docente en términos pedagógicos y didácticos. En consecuencia, se eligieron alumnos que cursaran el penúltimo semestre de la licenciatura en Pedagogía en la UNAM.

c. Aplicación del cuestionario a 25 alumnos que conformaron la muestra.

d. Elaboración de categoría de análisis con base en los referentes teóricos y los resultados obtenidos de la encuesta.

e. Codificación de los resultados con base en las categorías de análisis elaboradas para tal propósito: dimensión pedagógica, dimensión ética, capacidades pedagógicas y didácticas, cualidades profesionales del docente, dominio del conocimiento, prácticas educativas de apoyo y ayuda al aprendizaje, vínculo ético, valores.

f. Análisis e interpretación de los resultados desde la perspectiva de la ética docente y de la ética del cuidado.

\section{Resultados}

Los resultados de la encuesta sobre la ética de la docencia y de la enseñanza muestran que en un buen docente se articulan dos dimensiones: la pedagógica (25\%) y la ética (75\%). Estas dos dimensiones se representan en la tabla 1.

Tabla 1. Dimensiones de un buen docente

\begin{tabular}{|l||c||c||}
\hline & Respuestas & $\mathbf{\%}$ \\
\hline \hline Dimensión ética de un buen docente & 92 & $75 \%$ \\
\hline Dimensión pedagógica de un buen docente & 31 & $25 \%$ \\
\hline \hline Total respuestas & $\mathbf{1 2 3}$ & $\mathbf{1 0 0} \%$ \\
\hline \hline $\begin{array}{l}\text { Fuente: Ibarra, } R \text {. G. (2013). Encuesta sobre la ética de la docencia y de la enseñanza } \\
\text { aplicada a alumnos de la Licenciatura en Pedagogía de la UNAM }\end{array}$ \\
\hline
\end{tabular}

En la tabla 1 se evidencia que el grupo de estudiantes encuestados situaron a la dimensión ética (75\%) por encima de la dimensión pedagógica $(25 \%)$ de un buen docente. Al respecto, vale acotar que en la dimensión pedagógica se agruparon las respuestas que engloban al conjunto de capacidades pedagógicas y didácticas, mientras que en la dimensión ética se conjuntaron las respuestas que se refieren a las actitudes y acciones de apoyo y 


\section{Revista Iberoamericana \\ de las Ciencias Sociales y \\ Humanísticas}

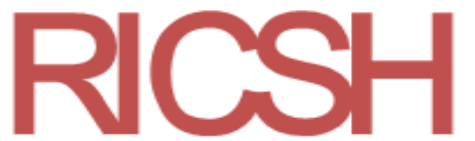

ISSN: 2395 - 7972

acompañamiento que puede ofrecer el docente al alumno en su proceso de aprendizaje. La separación de los resultados en estas dos dimensiones es solo con fines de análisis para mostrar sus diferencias y su complementariedad en el proceso de enseñanza y aprendizaje.

La dimensión pedagógica se asocia con que el docente es competente para llevar a cabo la enseñanza porque cuenta con las competencias pedagógicas y didácticas, mientras que la dimensión ética expresa la forma como se realiza la práctica de la enseñanza, es decir, se articula lo pedagógico y lo ético ya sea para facilitar el aprendizaje o solo para transmitir los conocimientos.

Estas dimensiones enseñan que para este grupo de estudiantes un buen docente adquiere este calificativo no solo porque cuenta con las capacidades profesionales para la enseñanza, sino también por las prácticas educativas y las actitudes éticas mediante las cuales contribuye al aprendizaje de los alumnos. Asimismo, es importante señalar que la dimensión ética también incluye los valores que los estudiantes consideran que distinguen a un buen docente, pero estos valores se muestran y analizan en la tabla 4.

Tabla 2. Dimensión pedagógica de un buen docente

\begin{tabular}{|l||c|c|}
\hline \hline & Respuestas & \% \\
\hline \hline $\begin{array}{l}\text { Competencias pedagógica y } \\
\text { didáctica }\end{array}$ & 14 & $44 \%$ \\
\hline \hline Cualidades de un buen docente & 12 & $38 \%$ \\
\hline \hline Dominio de contenidos & 6 & $19 \%$ \\
\hline \hline Total respuestas & $\mathbf{3 2}$ & $\mathbf{1 0 0} \%$ \\
\hline \hline $\begin{array}{l}\text { Fuente: Ibarra R. G. (2013). Encuesta sobre la ética de la } \\
\text { docencia y de la enseñanza aplicada a alumnos de la Licenciatura } \\
\text { en Pedagogía de la UNAM }\end{array}$ \\
\hline
\end{tabular}

En la tabla 2 se observa que los alumnos situaron en primer lugar a las competencias pedagógicas y didácticas (44 \%), seguidas de las cualidades de un buen docente (38 \%) y por último el dominio de los contenidos (19\%).

En el rubro de las competencias pedagógicas y didácticas se agruparon las respuestas que se refieren a las estrategias de enseñanza y a las técnicas didácticas mediante las cuales el docente transmite los contenidos o conocimientos. Los resultados exponen que para este grupo de estudiantes son más importantes el conjunto de elementos que integran la planificación y organización de la enseñanza que el dominio de los contenidos por parte del docente. Estos resultados se pueden considerar como un indicador de los cambios indispensables para la práctica docente, puesto que ha predominado la visión de que un buen 


\section{Revista Iberoamericana \\ de las Ciencias Sociales y \\ Humanísticas}

ISSN: $2395-7972$

docente es aquel profesionista que domina los conocimientos que transmite. Sin embargo, los resultados indican el valor que están adquiriendo los procesos y recursos pedagógicos y didácticos para la enseñanza debido a que constituyen los medios a través de los cuales los alumnos pueden acceder y asimilar los conocimientos que se imparten.

De acuerdo con la tabla 2, las cualidades de un buen docente fueron situadas en segundo orden de importancia. Este rubro lo componen aquellas respuestas que destacan atributos del docente vinculados con la actividad de la enseñanza, como son "organización”, "flexibilidad", "iniciativa", "dinamismo" y por supuesto "actualización permanente", por mencionar algunas de estas respuestas. Como puede advertirse, estos atributos no son rasgos del carácter o personalidad del docente, sino que son nuevas y novedosas propiedades de un docente que surgen ante las transformaciones que está experimentando la sociedad por el impacto de la innovación y el cambio en todos sus ámbitos. La flexibilidad implica la comprensión y la tolerancia, y no el apego a normas estrictas que impiden la evolución, mientras que el dinamismo es lo opuesto a la monotonía, por lo que en términos pedagógicos se refiere más al interés, esmero, dedicación y eficacia en la enseñanza.

Atendiendo a los resultados de la tabla 2, el dominio de contenidos fue ubicado en el tercer lugar con un porcentaje menor (19\%). Estos resultados permiten plantear que la concepción tradicional del buen docente comienza a ser desplazada por una visión innovadora, donde adquiere relevancia la renovación y diversificación de las capacidades de enseñanza y otros atributos, como la organización, flexibilidad para adaptarse a las condiciones y circunstancias educativas que se van presentando día a día, propiciar y fomentar el aprendizaje, así como la motivación para alcanzarlo. Todo esto es contrario a la concepción convencional y conservadora de la docencia, donde se destaca como el rasgo principal la posesión de conocimientos y como estrategia de enseñanza la exposición por parte del docente.

Tabla 3. Dimensión ética de un buen docente

\begin{tabular}{|l|c|c||}
\hline & Respuestas & \% \\
\hline \hline Acciones de ayuda y apoyo & 44 & $48 \%$ \\
\hline \hline Vínculo ético & 28 & $30 \%$ \\
\hline Valores & 20 & $22 \%$ \\
\hline \hline Total respuestas & $\mathbf{9 2}$ & $\mathbf{1 0 0 \%}$ \\
\hline \hline $\begin{array}{l}\text { Fuente: Ibarra R. G. (2013). Encuesta sobre la ética de la } \\
\text { docencia y de la enseñanza aplicada a alumnos de la Licenciatura } \\
\text { en Pedagogía de la UNAM }\end{array}$ \\
\hline
\end{tabular}




\section{Revista Iberoamericana \\ de las Ciencias Sociales y \\ Humanísticas}

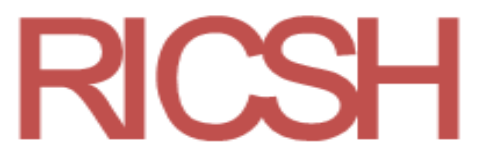

ISSN: 2395 - 7972

La tabla 3 muestra los componentes de la dimensión ética: acciones de ayuda y apoyo por parte del docente (48 \%), vínculo ético en el proceso de enseñanza y aprendizaje (30 \%) y valores (22\%). El rubro de las acciones de ayuda y apoyo fue situado como el rasgo más importante de un buen docente. Estos resultados se analizaron en el marco de la concepción de la docencia como una profesión de ayuda, lo cual permitió comprender este tipo de acciones como actividades propias de la enseñanza mediante las cuales los alumnos experimentan el acompañamiento del docente en su proceso de enseñanza. Muestra de ello son las siguientes respuestas: "proporcionar retroalimentación tanto en la clase como en los trabajos presentados", "resolver y aclarar dudas", "construir un clima de confianza en el aula", "tomar en cuenta la opinión de los alumnos", "mostrar interés por el aprendizaje de los alumnos", "promover la participación de los alumnos en la clase", "motivar a los alumnos", "estimular el aprendizaje de manera colaborativa".

Desde esta perspectiva, tales acciones no se refieren a la conducta del docente, sino a la asistencia pedagógica concreta a través de la cual se contribuye a favorecer el aprendizaje de los alumnos. La colaboración del docente en el aprendizaje de ellos se puede considerar como actividades propias de la docencia que en ocasiones pasan inadvertidas, pero que fueron altamente valoradas por el grupo de alumnos encuestados.

Las actividades pedagógicas y educativas de apoyo - lejos de crear una dependencia de los alumnos hacia el docente - representan la forma específica como el docente puede coadyuvar a que el alumno vaya logrando su aprendizaje por sí mismo. Estas acciones se ubicaron en el rubro de la dimensión ética de un buen docente porque la atención, el acompañamiento, la asistencia, el cuidado y la colaboración por parte del docente son expresiones de la responsabilidad que le corresponde asumir en el proceso de enseñanza y aprendizaje.

La tabla 3 da cuenta que el grupo de estudiantes encuestados colocaron en segundo lugar de importancia al vínculo ético en el proceso de enseñanza y aprendizaje (30 \%). Estos resultados se analizaron desde la perspectiva de la ética del cuidado para dar cuenta de la importancia que tiene el tipo de relación educativa en el proceso de enseñanza y aprendizaje. Como señalamos en los referentes teóricos para esta ética, la ayuda y el apoyo que puede

ofrecer el docente se puede concretar mediante una relación educativa que se estructure como un vínculo ético. Es decir, requiere que el docente tome en cuenta la relación asimétrica que 


\section{Revista Iberoamericana \\ de las Ciencias Sociales y \\ Humanísticas}

ISSN: $2395-7972$

sitúa a los alumnos en una condición educativa diferente a la del docente porque no cuentan con los conocimientos que necesitan para desarrollarse.

Esta situación inherente a la relación educativa constituye la base para que el docente pueda tener la actitud y disposición ética para apoyar y cuidar el aprendizaje de los alumnos mediante la construcción de la relación educativa como un vínculo ético. Ejemplos de este tipo de actitudes son las siguientes respuestas: "amabilidad", "accesibilidad", "respeto", "empatía con los alumnos", "brindar confianza a los alumnos", "paciente", "abierto al diálogo", "que sepa escuchar a los alumnos", "tolerante", "no sentir superioridad ante el grupo", “apertura a las ideas de los demás", "amistoso", “alegre”.

Estos hallazgos coinciden con los obtenidos por Narinsamy y Logeswaran (citados por Comins, 2017), quienes dan cuenta del perfil de un caring teacher. Comins (2017) traduce este término como "profesor cuidadoso y atento" y expone los seis componentes de este perfil: 1) pone en valor las capacidades del estudiantado, 2) se preocupa por su bienestar, 3) muestra paciencia, 4) escucha al estudiantado, 5) trata al estudiante de manera justa, 6) empatiza con el estudiantado (cursivas de la autora) (Comins, 2017, p. 158).

Tres componentes del perfil del caring teacher coinciden con tres rasgos de un buen docente agrupados en el rubro del vínculo ético paciencia, empatía y escucha a los alumnos. Los valores de un buen docente representan $22 \%$ de las respuestas. Estos valores se exponen en la siguiente tabla, al lado de los valores para la formación docente que propusieron el grupo de estudiantes encuestados.

Tabla 4. Valores del docente

\begin{tabular}{|c|c|c|c|c|c|}
\hline \multicolumn{3}{|c|}{ Valores para la formación docente } & \multicolumn{3}{|c|}{ Valores de un buen docente } \\
\hline 1.-Responsabilidad & 16 & $28 \%$ & Responsabilidad & 7 & $37 \%$ \\
\hline 2.- Respeto & 14 & $25 \%$ & Compromiso & 6 & $32 \%$ \\
\hline 3.-Compromiso & 10 & $18 \%$ & Respeto & 5 & $26 \%$ \\
\hline 4.- Tolerancia & 9 & $16 \%$ & Tolerancia & 1 & $5 \%$ \\
\hline 5.- Honestidad & 8 & $14 \%$ & Honestidad & 1 & $37 \%$ \\
\hline Total valores & 57 & $100 \%$ & Total valores & 19 & $100 \%$ \\
\hline
\end{tabular}

En la tabla 4 se muestran los valores que agrupan dos tipos de respuesta: los valores referidos como rasgos de un buen docente y los valores que se plantean para la futura formación de los docentes. De cada grupo de valores se eligieron los cinco que obtuvieron el 


\section{Revista Iberoamericana \\ de las Ciencias Sociales y \\ Humanísticas}

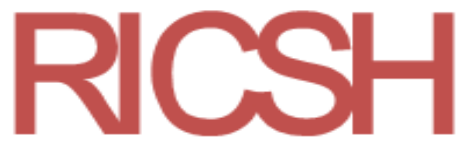

ISSN: $2395-7972$

mayor número de respuestas. Con este mismo criterio metodológicos se eligieron los cinco valores propuestos para a la formación docente.

El valor que agrupó el mayor número de respuestas fue la responsabilidad. Con base en esta estimación, es posible considerar que para este grupo de alumnos es el criterio más significativo o relevante de un docente, el cual se puede asociar con el cumplimiento de las obligaciones profesionales del docente. Sin embargo, desde la concepción de la docencia como una profesión de ayuda, más que un deber, se considera la expresión de las actividades positivas que realiza el docente a favor del aprendizaje de los alumnos (Altarejos, 2003).

Desde la perspectiva de la ética del cuidado, la responsabilidad se entiende como la respuesta del docente a las necesidades educativas de los alumnos (Noddings, 2009). Un aspecto que se destaca en estos resultados es que los valores del respeto y el compromiso fueron tomados en cuenta como rasgos de un buen docente y como valores para la formación docente, pero la diferencia se establece al detectar el lugar en el que fueron situados en cada uno de estos rubros.

La tabla 4 expone que en el apartado de los rasgos de un buen docente el valor del respeto fue colocado en primer término, y en segundo lugar el valor del compromiso. Este orden se invirtió en el rubro de los valores para la formación docente.

El significado dominante del valor del compromiso es la realización de las actividades de enseñanza y aprendizaje como un deber u obligación profesional del docente. Pero desde la concepción de la docencia como profesión de ayuda representa la exigencia moral de desempeñar la enseñanza buscando el beneficio de los alumnos (Altarejos, 2003). Este valor también implica desarrollar al máximo las capacidades y competencias pedagógicas y didácticas para que los alumnos alcancen el aprendizaje.

El respeto es un valor positivo que involucra la consideración y el reconocimiento del docente hacia los alumnos como sujetos educativos que también dotan de sentido el proceso educativo. Si bien para llevar a cabo la enseñanza es necesario el docente porque tiene los conocimientos y capacidades profesionales que se requieren para desarrollar el proceso educativo, estas actividades adquieren sentido por la presencia y participación de los alumnos. El valor del respeto fractura la concepción de la docencia que empodera al docente como el sujeto educativo clave del proceso educativo para dar paso a una visión donde los alumnos adquieren relevancia debido a que dotan de significado a la enseñanza, puesto que la razón de ser de esta actividad es el aprendizaje de los alumnos. 


\section{Revista Iberoamericana \\ de las Ciencias Sociales y \\ Humanísticas}

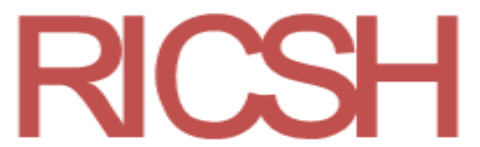

ISSN: 2395 - 7972

Desde esta perspectiva, este valor se puede desarrollar en actitudes y acciones concretas como el acompañamiento, la empatía, la tolerancia, la confianza y la solidaridad, las cuales constituyen actitudes propias del vínculo ético que se puede construir en la relación educativa.

De acuerdo con la tabla 4, la tolerancia aparece en el cuarto lugar en ambos grupos de valores. Este es un valor moral que involucra la comprensión de una situación educativa diferente que requiere la apertura por parte del docente para solucionar de manera positiva esta nueva circunstancia que se presenta en su práctica cotidiana. La tolerancia también permite reconocer y comprender las condiciones educativas distintas que tienen los alumnos, las cuales demandan atención y respuestas educativas diferentes. Las actitudes mediante las cuales se expresa la tolerancia son, entre otras, flexibilidad, paciencia, amabilidad y cordialidad.

La honestidad ocupa el quinto lugar y el significado dominante de este valor se refiere al actuar de manera correcta conforme a la verdad y apegado a deberes y normas morales. Pero desde una perspectiva ética, este valor va más allá de un comportamiento moral recto, porque comprende el buen obrar entendido como la realización de las actividades educativas con un sentido ético. Es decir, buscando el beneficio de los alumnos debido a la importancia que tiene la educación no solo para los estudiantes, sino para la sociedad en su conjunto. Esto determina que, en término éticos, no caben las actividades de enseñanza que se desarrollan solo para el provecho personal de los docentes. La honestidad también permite establecer relaciones de cercanía y certeza porque están basadas en el respeto mutuo, la confianza y la sinceridad. Desde esta perspectiva, el valor de la honestidad es clave para construir la relación educativa como un vínculo ético.

\section{Discusión}

Como señalamos anteriormente, el tema del buen docente ha sido motivo de diferentes investigaciones, como las realizadas por Corona (2008), Giné (2009), Casero (2010), Cabalín, Navarro, Zamora y San Martín (2010) y Alonso (2019), autores que se han destacado porque han tenido como perspectiva de análisis a la pedagogía y a la educación. Esas indagaciones reportan rasgos y características de un buen docente que también están presentes en este estudio de la ética docente. La diferencia estriba en que el enfoque de la ética docente permite distinguir los atributos pedagógicos y educativos, así como los rasgos 


\section{Revista Iberoamericana \\ de las Ciencias Sociales y \\ Humanísticas}

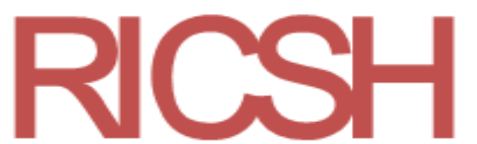

ISSN: $2395-7972$

éticos de un buen docente. Por ello, en este estudio se considera que en un buen docente se integran dos dimensiones: la pedagógica y la ética.

Estas investigaciones, por otra parte, ofrecen resultados que se pueden comprender como propios de la dimensión ética, pero que desde el enfoque pedagógico y educativo adquieren otro significado. Así, en Corona (2008) los atributos éticos se conciben bajo la categoría de lo social. En Casero (2010) quedan englobados en la categoría de variables personales del profesor, mientras que en la investigación de Cabalín, Navarro, Zamora y San Martín (2010) se conciben con la categoría del saber ser y saber convivir, y en el estudio de Alonso (2019) como cualidades personales del buen docente. Los estudios muestran que esas características del buen docente fueron altamente valoradas por los estudiantes encuestados para definir los rasgos de un buen docente.

El enfoque de la ética docente permite comprender los rasgos éticos de un buen docente no como atributos personales o competencias profesionales, sino como acciones y prácticas pedagógicas que dotan de sentido al proceso de enseñanza porque están enfocadas a colaborar en el aprendizaje de los alumnos. Desde esta postura, la razón de ser de la enseñanza no reside tanto en la personalidad o el carácter del docente, sino en las acciones educativas que realice para contribuir a que los alumnos alcancen el aprendizaje esperado.

La dimensión ética no pretende moldear los rasgos personales del docente, sino que apunta a la toma de conciencia por parte del docente del propósito u objetivo esencial que tiene la enseñanza, es decir, que los alumnos aprendan, para lo cual debe desarrollar las acciones y actitudes pedagógica y educativas de apoyo y ayuda necesarias.

Sin embargo, para alcanzar el aprendizaje de los alumnos no es suficiente que el docente tenga la disposición ética y lleve a cabo prácticas educativas de apoyo y colaboración; también debe contar con los conocimientos y capacidades profesionales para atender y solucionar las necesidades educativas diferentes que tienen los alumnos. Por ello, este estudio integra la dimensión pedagógica como un componente de la ética docente. 


\section{Revista Iberoamericana \\ de las Ciencias Sociales y \\ Humanísticas}

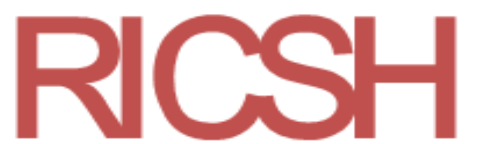

ISSN: $2395-7972$

\section{Conclusiones}

Los resultados obtenidos con la encuesta contribuyeron a conocer que en la ética docente confluyen dos dimensiones: la pedagógica y la ética. En el marco de esta ética, los atributos pedagógicos del buen docente son los que le otorgan la calificación para prestar el servicio que le corresponde proporcionar a la sociedad. Estos atributos respaldan la responsabilidad inherente del docente porque indican que está preparado y es competente para realizar la enseñanza de manera eficiente y con calidad. Las competencias profesionales son la base necesaria a partir de las cual el docente puede desarrollar la práctica de enseñanza con un sentido ético, es decir, en beneficio del aprendizaje del alumno.

Ahora bien, estos resultados también muestran que el buen docente adquiere este calificativo por un conjunto de actividades y actitudes de ayuda y apoyo agrupadas en la dimensión ética. Como se expuso anteriormente, estas prácticas educativas obtuvieron el mayor número de respuestas ( $75 \%$ ), por lo que fueron situadas por encima de la dimensión pedagógica del buen docente. Con base en esta estimación, es posible considerar que en este estudio el núcleo de la ética docente es este conjunto de prácticas educativas éticas que adquieren este significado porque se realizan a favor del aprendizaje de los alumnos.

El corazón de la ética docente son estas prácticas educativas porque son el vehículo para que el docente enfoque sus capacidades profesionales para ofrecer el bien que le corresponde prestar. Estos resultados dan cuenta de que la ética docente se expresa en el desarrollo del proceso de enseñanza cuando el docente toma en cuenta y atiende las necesidades educativas de los alumnos haciendo uso de sus competencias profesionales para apoyarlos y ayudarlos a conseguir el aprendizaje. Esto es posible si el docente comprende su labor como una profesión de ayuda, y no como una ocupación que proporciona un servicio más a la sociedad.

La dimensión ética del buen docente ofrece resultados para entender las acciones y prácticas educativas concretas mediante las cuales la ética docente se puede desarrollar en el proceso de enseñanza y aprendizaje. La perspectiva de la ética del cuidado permitió comprender estas actividades como acciones de ayuda, cuidado y colaboración que puede brindar el docente a los alumnos, pero que en sí mismas son prácticas pedagógicas y educativas que expresan la atención y respuesta del docente a las diferentes necesidades formativas de los alumnos. 


\section{Revista Iberoamericana \\ de las Ciencias Sociales y \\ Humanísticas}

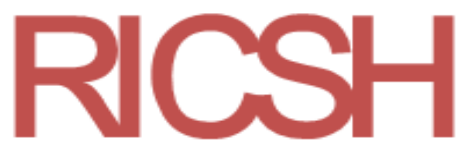

ISSN: 2395 - 7972

La aportación de este estudio comprende dos aspectos. La dimensión pedagógica del buen docente da cuenta de la importancia y relevancia que han adquirido las capacidades pedagógicas y didácticas para la enseñanza porque los resultados indican que el aprendizaje depende en gran medida de estas competencias profesionales. El grupo de alumnos encuestados hace patente la necesidad de diversificar las estrategias de enseñanza e incorporar nuevas y diferentes técnicas didácticas para el aprendizaje. En términos de la ética docente, los resultados remiten a la responsabilidad inherente de la profesión docente, entendida como el deber o la obligación de mantener actualizados sus conocimientos y competencias pedagógicas para llevar a cabo una mejor enseñanza.

La contribución de la dimensión ética es que pone en el centro del proceso de enseñanza a la relación educativa mostrando el valor que tiene para que los alumnos alcancen el aprendizaje. Esto porque el grupo de alumnos encuestados situó a la dimensión ética por encima de la pedagógica. Con ello, estos resultados muestran que el modelo de la docencia tradicional — sustentada en el dominio de los contenidos - ha llegado a su límite y que actualmente es más relevante el tipo de relación educativa que se establezca con los alumnos, así como las actividades educativas mediante las cuales se apoya el aprendizaje. El paradigma dominante de la docencia no contempla la asistencia, el acompañamiento y el respaldo que puede ofrecer el docente a los alumnos como prácticas pedagógicas porque el aprendizaje se concibe como el resultado del conocimiento que transmite el docente a través de la clase magistral.

La dimensión ética, en otras palabras, no se reduce a un comportamiento apegado a normas y valores morales, sino que es una ética práctica que se desarrolla mediante actividades pedagógicas y educativas concretas cuyo sentido es apoyar y ayudar a los alumnos en su aprendizaje. Los resultados que engloba esta dimensión exponen un conjunto de estas actividades educativas específicas, las cuales muestran cómo y en qué consiste la realización de la ética docente en el proceso educativo.

El estudio de la ética docente que se presenta en este artículo muestra que esta ética no es un planteamiento teórico abstracto y alejado de la realidad, sino que está presente en el contexto educativo como una necesidad o ideal que tienen los alumnos. Los resultados de este estudio indican que la ética docente puede tener cabida en el ámbito educativa porque contribuye a potenciar las capacidades de aprendizaje de los futuros profesionistas. 


\section{Revista Iberoamericana \\ de las Ciencias Sociales y \\ Humanísticas}

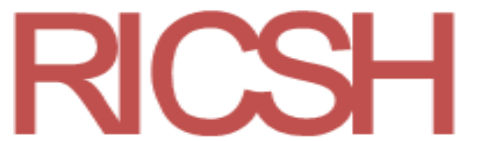

ISSN: $2395-7972$

No obstante, se puede considerar como una limitación de este estudio que los resultados obtenidos no se pueden generalizar porque la encuesta se aplicó a un grupo de estudiantes de pedagogía. Aun así, muestran la pertinencia de continuar desarrollando esta temática de investigación en otros espacios educativos para contribuir al avance de la construcción de la ética docente a nivel universitario.

\section{Futuras líneas de investigación}

Este trabajo permitió conocer e identificar las dimensiones de la ética docente. Empero, debido a su carácter exploratorio, no se profundizó en las causas que afectan o inciden en la realización de la ética docente en el aula. Por ello, resulta conveniente que a futuro se realicen investigaciones que permitan conocer cuáles son los factores o elementos que impactan en el desarrollo de esta ética. Esto porque los docentes llevan a cabo su práctica de enseñanza en un marco institucional y pedagógico que determina sus acciones.

Finalmente, se puede seguir indagando en cuáles son las competencias de enseñanza que requieren los profesores para lograr el aprendizaje y cuál es el tipo de relación educativa que mejor favorece ese objetivo.

\section{Referencias}

Altarejos, F. (2003). La docencia como profesión asistencial. En Altarejos, F., Ibáñez-Martín, J., Jordán, J. y Jover, G. (coords.), Ética docente. Barcelona: Ariel.

Alonso, M. P (2019). El perfil del buen docente universitario según la valoración de alumnos del magisterio y psicopedagogía. Perfiles Educativos, 41(164). Recuperado de http://perfileseducativos.unam.mx/iisue_pe/index.php/perfiles/article/view/58906/52 417

Cabalín, S. D., Navarro, H. N., Zamora, S. J. y San Martín, G. S. (2010). Concepción de estudiantes y docentes del buen profesor universitario. Facultad de Medicina de la Universidad de la Frontera. Int. J. Morphol, 28(1), Recuperado de https://scielo.conicyt.cl/scielo.php?script=sci_arttext\&pid=S0717-

95022010000100042 


\section{Revista Iberoamericana \\ de las Ciencias Sociales y \\ Humanísticas}

ISSN: $2395-7972$

Casero, M. A. (2010). ¿Cómo es el buen profesor universitario según el alumnado? Revista Española de Pedagogía, 68(246). Recuperado de https://revistadepedagogia.org/wpcontent/uploads/2010/06/246-002.pdf

Comins, M. I. (2017). ¿Hacia qué modo-de-ser- en el mundo? Por una pedagogía del cuidar. Documentación Social, (187), 145-160.

Corona, A. C. (2008). ¿Qué hace al buen maestro? La visión de los estudiantes de ciencias físico matemáticas. Lat. Am.J. Phys. Educ., 2(2). Recuperado de http://www.lajpe.org/may08/08_Adrian_Corona.pdf

Cortina, A. (2000). Presentación. El sentido de las profesiones. En Cortina, A. y Conill, J. (dirs.), 10 palabras clave en ética de las profesiones. Navarra: Verbo Divino.

García, A. M. y García, G. J. (2012). Deontología para profesionales de la educación. Madrid: Editorial Universitaria Ramón Arce/UNED.

García, L. R, Jover, O. G. y Escámez, S. J. (2010). Ética profesional docente. Madrid: Síntesis.

García, M. F. (1998). La ética del profesorado. En García Moriyón, F. (ed.), Crecimiento moral y filosofía para niños (pp. 293-310). Bilbao: Desclée De Brouwer.

Giné, N. (2009). Cómo mejorar la docencia universitaria: el punto de vista del estudiantado. Revista Complutense de Educación, 20(1). Recuperado de https://revistas.ucm.es/index.php/RCED/article/view/RCED0909120117A/15402

Hirsch, A. A, (2005). Construcción de una escala de actitudes sobre la ética profesional. Revista Electrónica de Investigación Educativa, 7(1).

Hortal, A. A. (2002). Ética general de las profesiones. Bilbao: Desclée De Brouwer.

Ibarra, R. G. (2013). Encuesta sobre la ética de la docencia y de la enseñanza realizada a alumnas(os) de $8^{\circ}$ semestres de la licenciatura en Pedagogía de la Facultad de Filosofía y Letras de la UNAM. México: UNAM.

Ibarra, R. G. (2018). Educación y ética del cuidado. En Santillán Campos, F. (coord.), La investigación en las instituciones de educación superior. Guadalajara, México: Centro de Estudios e Investigaciones para el Desarrollo Docente (CENID). 
Revista Iberoamericana

de las Ciencias Sociales y

Humanísticas

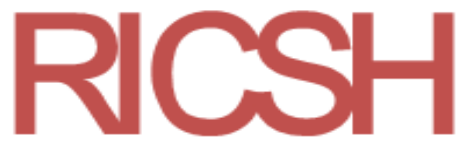

ISSN: 2395 - 7972

López-Roldán, P. y Fachelli, S. (2015). Metodología de investigación social cuantitativa.

Barcelona: Universidad Autónoma de Barcelona. Recuperado de http://ddd.uab.cat/record/129382

Martínez, N. E. (2010). Ética profesional de los profesores. Bilbao: Desclée De Brouwer.

Mesa, J. A (2004). Congreso de la educación desde las éticas del cuidado y la compasión. Bogotá: Universidad Javeriana.

Noddings, N. (2009). La educación moral. Propuesta alternativa para la educación del carácter. Buenos Aires: Amorrortu.

Weis, R. (2007). Programa de formación ética: desarrollo de una cultura de cuidado. Buenos Aires: Ediciones Novedades Educativas. 